\title{
On the Feasibilities of Using the Wavelet Analysis of Mueller Matrix Images of Biological Crystals
}

\author{
O. V. Dubolazov, ${ }^{1}$ A. G. Ushenko, ${ }^{1}$ V. T. Bachynsky, ${ }^{2}$ A. P. Peresunko, ${ }^{2}$ and O. Ya. Vanchulyak ${ }^{2}$ \\ ${ }^{1}$ Optics and Spectroscopy Department, Chernivtsi National University, 2 Kotsyubinsky Str., 58012, Chernivtsi, Ukraine \\ ${ }^{2}$ Department of Morbid Anatomy, Chernivtsi Medical University, 2 Theatral Sq, 58002, Chernivtsi, Ukraine
}

Correspondence should be addressed to A. G. Ushenko, yuriyu@gmail.com

Received 5 December 2009; Accepted 29 March 2010

Academic Editor: Oleg V. Angelsky

Copyright (C) 2010 O. V. Dubolazov et al. This is an open access article distributed under the Creative Commons Attribution License, which permits unrestricted use, distribution, and reproduction in any medium, provided the original work is properly cited.

\begin{abstract}
The efficiency of using the statistical and fractal analyses for distributions of wavelet coefficients for Mueller matrix images of biological crystal networks inherent to human tissues is theoretically grounded in this work. The authors found interrelations between statistical moments and power spectra for distributions of wavelet coefficients as well as orientation-phase changes in networks of biological crystals. Also determined are the criteria for statistical and fractal diagnostics of changes in the birefringent structure of biological crystal network, which corresponds to pathological changes in tissues.
\end{abstract}

\section{Introduction}

In recent years, laser diagnostics aimed at the structure of biological tissues efficiently use the model approach [1], in accordance with the tissues that are considered as two components: amorphous $\{A\}$ and optically anisotropic $\{F\}$ ones. Each of these components is characterized by intrinsic matrix operators

$$
\{A\}=\left\|\begin{array}{llll}
1 & 0 & 0 & 0 \\
0 & 1 & 0 & 0 \\
0 & 0 & 1 & 0 \\
0 & 0 & 0 & 1
\end{array}\right\| \cdot e^{-\tau l}
$$

where $\tau$ is the extinction coefficient inherent to the layer of biological tissue with the geometric thickness $l$

$$
\{F\}=\left\|\begin{array}{cccc}
1 & 0 & 0 & 0 \\
0 & f_{22} & f_{23} & f_{24} \\
0 & f_{32} & f_{33} & f_{34} \\
0 & f_{42} & f_{43} & f_{44}
\end{array}\right\|
$$

$$
=\left\|\begin{array}{cccc}
1 & 0 & 0 & 0 \\
0 & \cos ^{2} 2 \rho+\sin ^{2} 2 \rho \cdot \cos \delta & \cos 2 \rho \sin 2 \rho(1-\cos \delta) & \sin 2 \rho \sin \delta \\
0 & \cos 2 \rho \sin 2 \rho(1-\cos \delta) & \sin ^{2} 2 \rho+\cos ^{2} 2 \rho \cos \delta & \cos 2 \rho \sin \delta \\
0 & -\sin 2 \rho \sin \delta & -\cos 2 \rho \sin \delta & \cos \delta
\end{array}\right\|
$$

Here, $\rho$ is the orientation of a protein fibril in the architectonic network, the matter of which introduces the phase shift $\delta$ between orthogonal components of the laser wave amplitudes.

Topicality of this modeling is related with the possibility to apply the all-purpose Mueller matrix analysis to changes of polarization properties, which are caused by transformation of optical and geometric constitution of the anisotropic component (architectonic network of fibrils) in these biological objects [2-8]. Based on this model, there is the developed method for polarization differentiation of optical properties inherent to physiologically normal as well as pathologically changed biological tissues by using the wavelet analysis of local features observed in coordinate distributions of intensities in their coherent images.

This trend in polarization diagnostics got its development in investigations of a statistical and self-similar structure of Mueller-matrix images (MMIs) that are twodimensional distributions $f_{i k}(x, y)[9,10]$ describing biological tissues. So, in the approximation of single light 

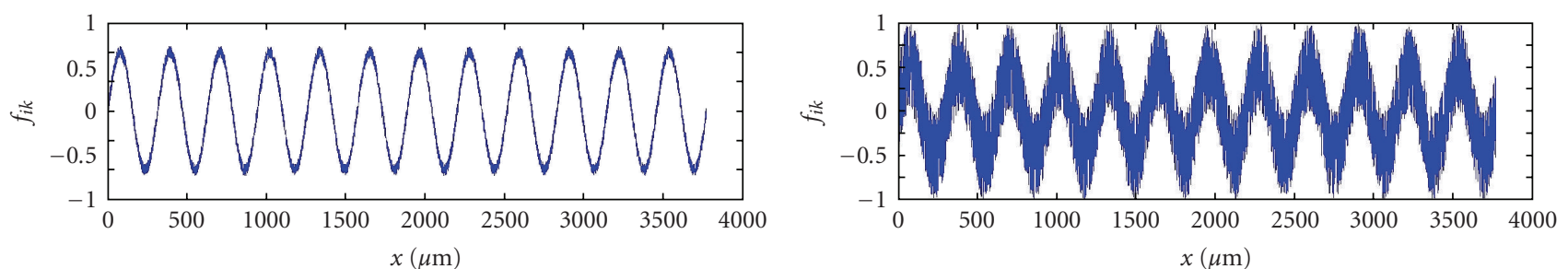

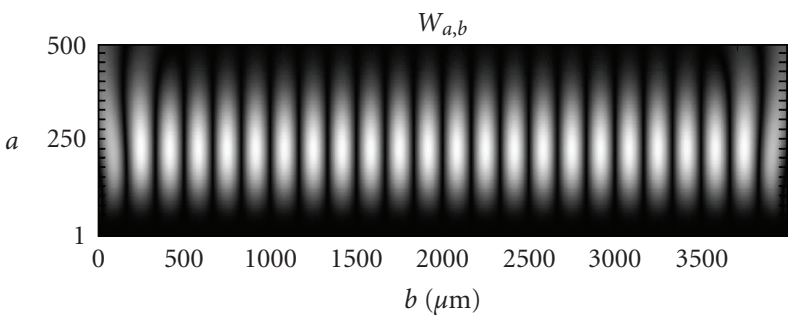

(a)

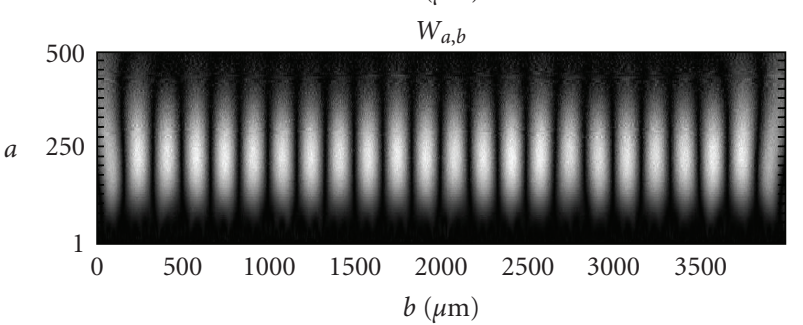

(b)
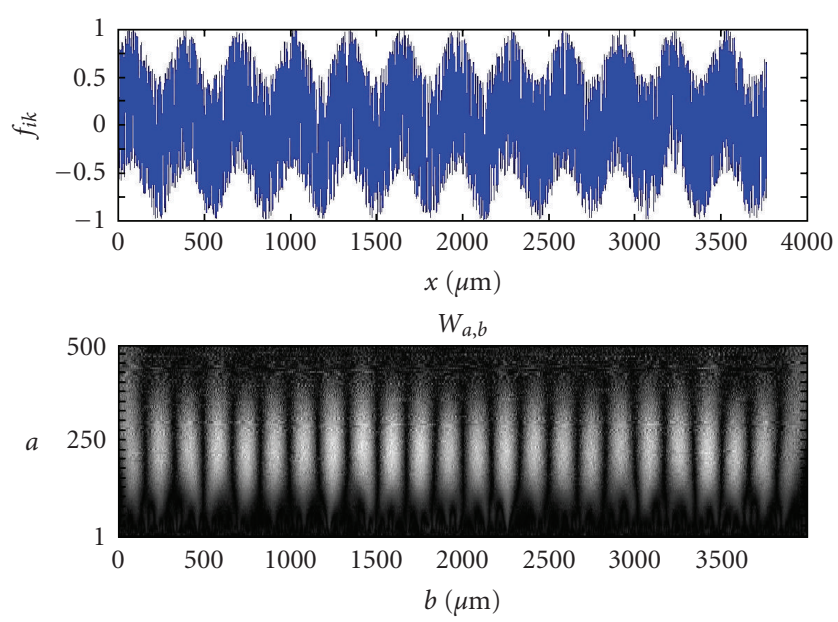

(c)

FIGURE 1: Wavelet coefficients $W_{a, b}$ of statistical and stochastic distributions for the matrix element $f_{i k}(x \equiv b)$. Commentaries are in the text.

scattering, there was found the interrelation between a set of statistical moments of the first to fourth orders $Z^{(j=1,2,3,4)}$ that characterize orientation $(\rho)$ and phase $(\delta)$ structures of birefringent architectonics inherent to biological tissues as well as a set of respective statistical moments for MMI [10-14]. It is ascertained that the coordinate distributions of matrix elements $f_{i k}(x, y)$ describing physiologically normal biological tissue possess a self-similar, fractal structure. MMIs of physiologically changed biological tissues are stochastic or statistical [11].

This work is aimed at studying the efficiency of the wavelet analysis in application to the local structure of MMI inherent to biological tissues with using statistical and fractal analyses of the obtained wavelet coefficient distributions for diagnostics of local changes in orientation-phase structure of their architectonic networks.

\section{Wavelet Analysis of Mueller-Matrix Images of Biological Tissues}

Wavelet transformation of MMI consisted of its expansion within a basis of definite scale changes and transfers of the soliton-like function (wavelet) [6]. The distribution of values for $f_{i k}$ elements of the Mueller matrix can be represented in the following form:

$$
f_{i k}(x)=\sum_{j, l=-\infty}^{\infty} q_{j l} v_{j l}(x) .
$$

Here, $f_{i k}(x)$ distribution belongs to the space $L^{2}(R)$ created by wavelets $v_{j l}$. The basis of this functional space can be constructed using scale transformations and transfers of 


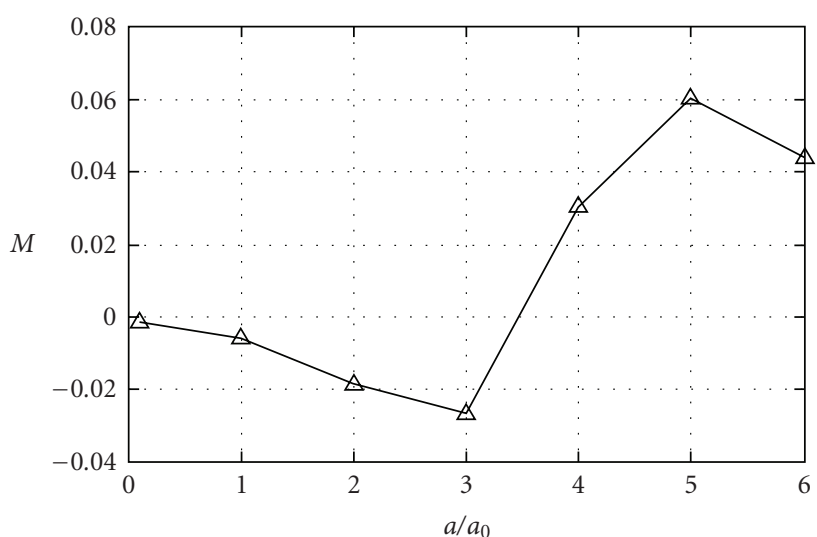

(a)

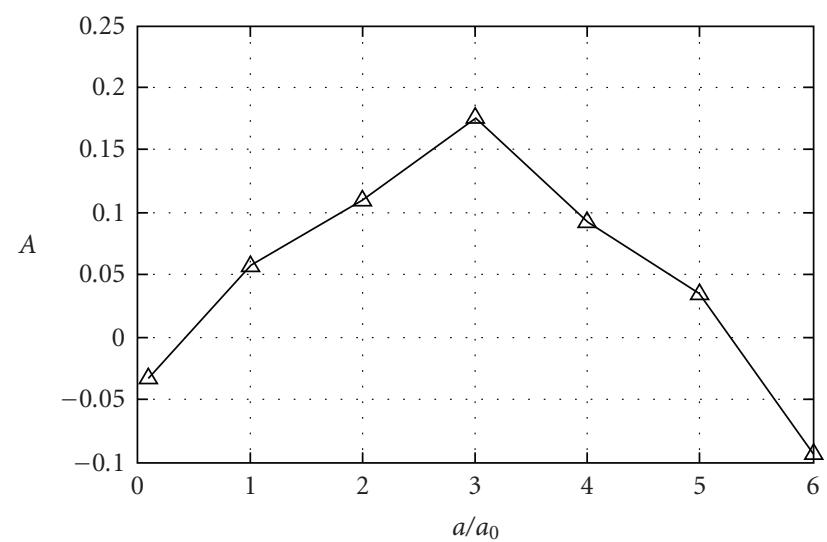

(c)

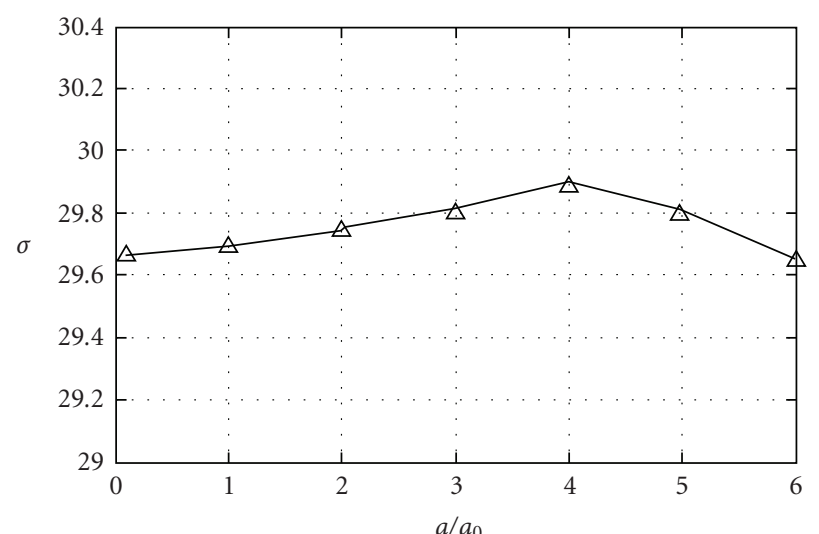

(b)

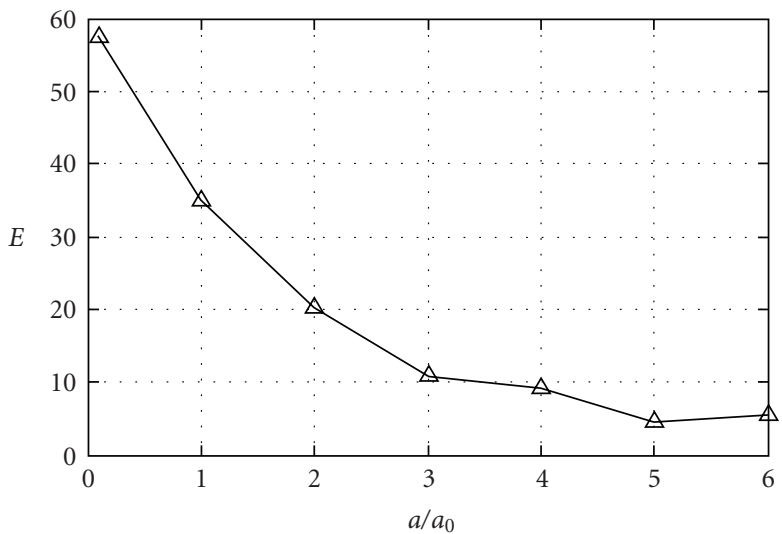

(d)

Figure 2: Mean value (a), dispersion (b), the skewness (c), and the kurtosis (d) of distributions inherent to wavelet coefficients $W_{a, b}\left(f_{i k}\right)$. Commentaries are in the text.

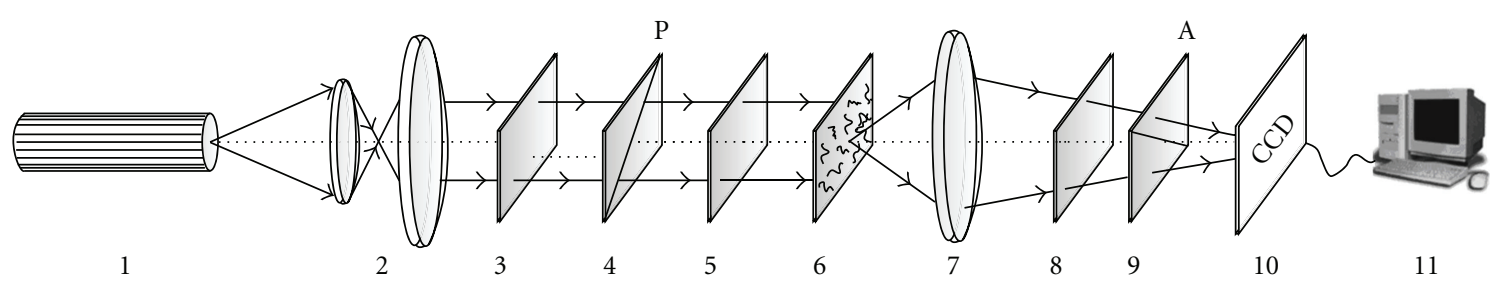

Figure 3: Optical scheme of polarimeter 1:He-Ne laser; 2:collimator; 3:stationary quarter-wave plates; 5, 8:mechanically movable quarterwave plates; 4, 9:polarizer and analyzer correspondingly; 6:object of investigation; 7:micro-objective; 10:CCD camera; 11:personal computer.

the wavelet $v_{j l}(x)$ with arbitrary values of basic parametersthe scaling coefficient $a$ and shift parameter $b$

$$
v_{a b}(x)=\left|a^{-1 / 2}\right| \xi\left(\frac{x-b}{a}\right) ; \quad a, b \in R ; \xi \in L^{2}(R) .
$$

Being based on it, the integral wavelet transformation takes a look at

$$
\begin{aligned}
{\left[W_{v} f_{i k}\right](a, b) } & =|a|^{-1 / 2} \int_{-\infty}^{\infty} f_{i k}(x) v^{*}\left(\frac{x-b}{a}\right) d x \\
& =\int_{-\infty}^{\infty} f_{i k}(x) v_{a b} *(x) d x
\end{aligned}
$$

Coefficients $q_{j l}=\left\langle f_{i k}, v_{j l}\right\rangle$ of the expansion (3) for the function $f_{i k}$ by wavelets can be defined via the following integral wavelet transformation:

$$
q_{j l}=\left[W_{v} f_{i k}\right]\left(\frac{1}{2^{j}}, \frac{l}{2^{j}}\right) .
$$

In our work, to analyze MMI we used the most widely spread soliton-like function MHAT ("Mexican hat", [6]) as a wavelet function. Such a function was effectively used in [15-19] for analysis of multiscale laser images of rough surfaces and histological sections of biological tissues. This case provides the possibility of comparative analysis of this 
TABLE 1: Log-log dependences of power spectra for the wavelet coefficients $W_{a, b}$ of statistical-stochastic distributions for $f_{i k}$ elements of the Mueller matrix describing single-axis biological crystals.

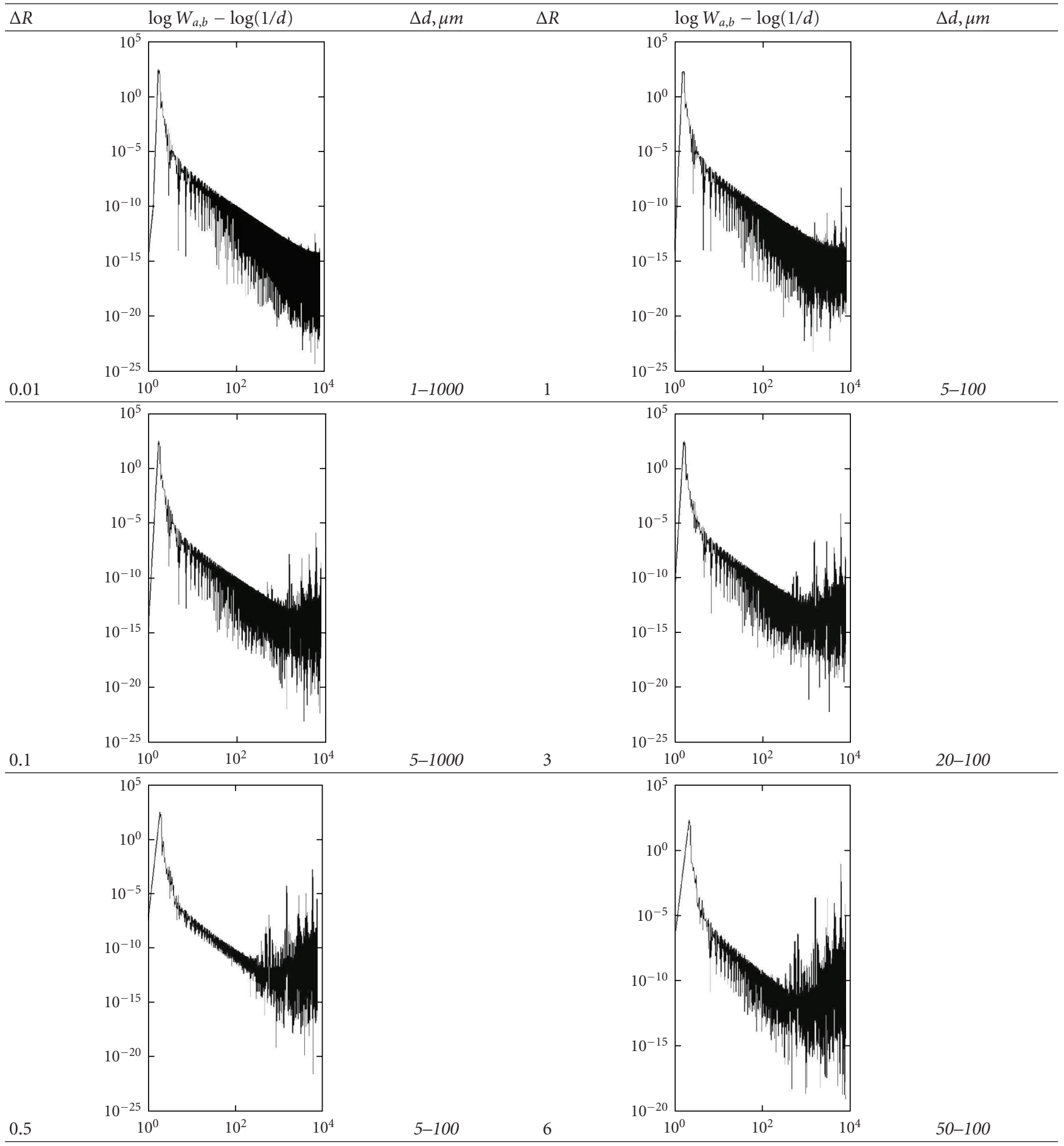

work with previous research of polarization inhomogeneous images of phase inhomogeneous layers. on the other hand, the task of selection of the most diagnostic appropriate function remains topical. However, this important task we consider as individual and it deserves the special research, which is not provided in our work.

\section{Computer Modeling the Efficiency of the Wavelet Analysis to Differentiate MMI of Birefringent Fibrils}

Birefringent architectonic networks of BT consist of a set of coaxial cylinder protein fibrils with a statistical distribution 


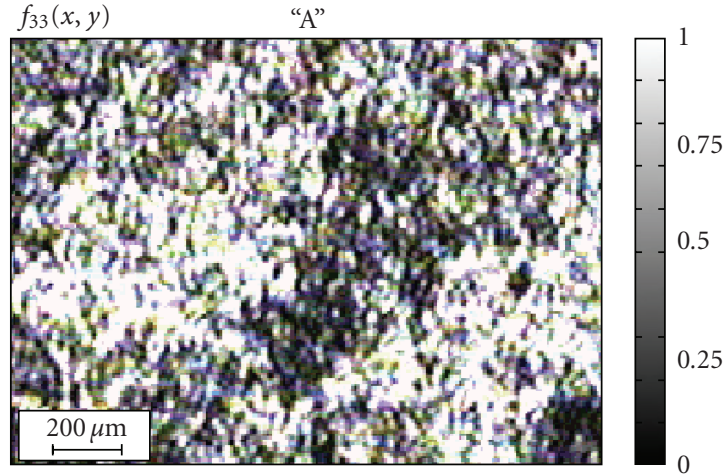

(a)

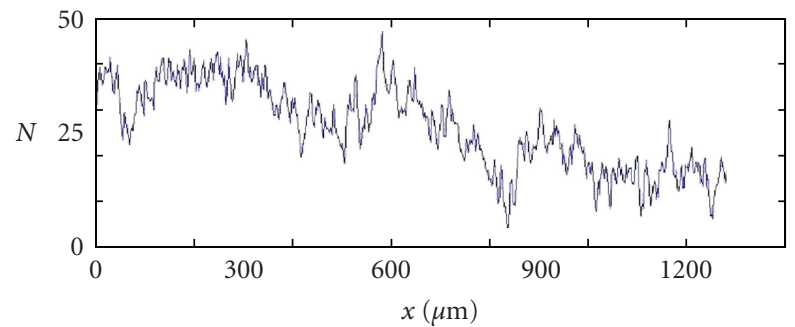

(c)

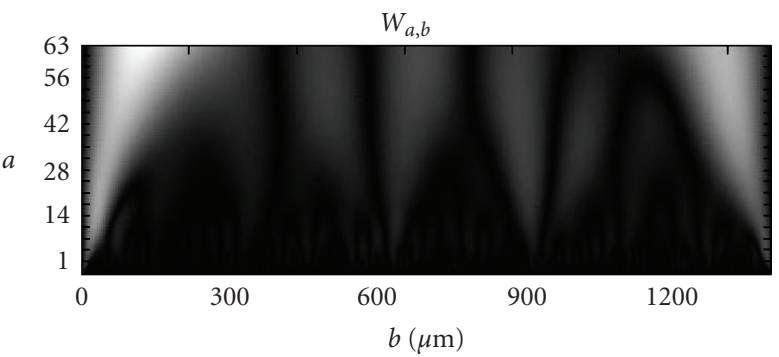

(e)

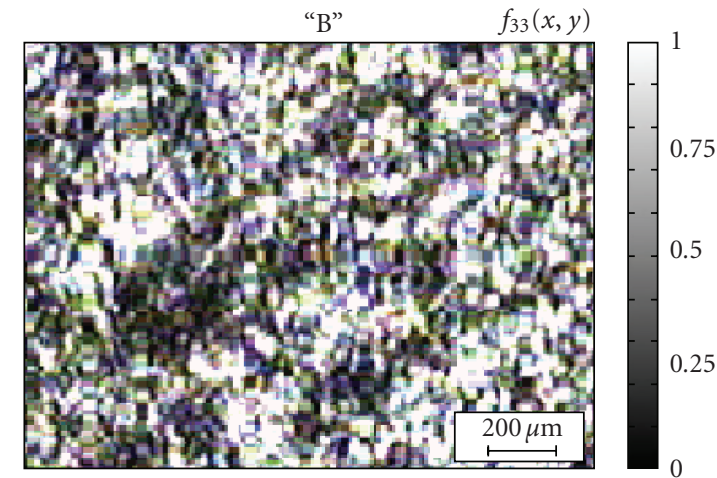

(b)

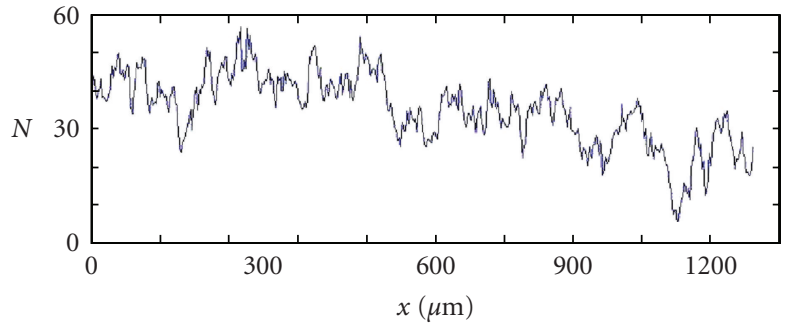

(d)

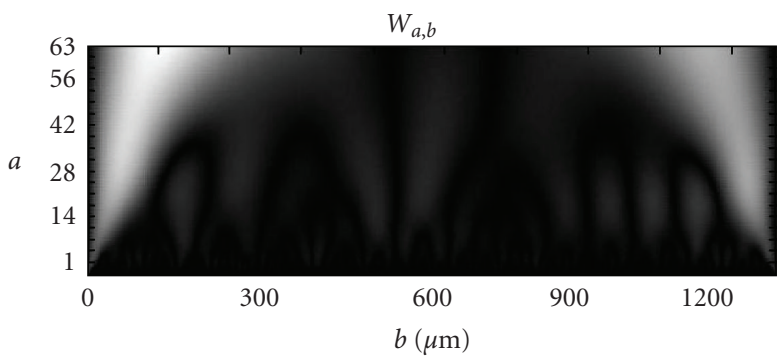

(f)

FIgURE 4: MMI of the $f_{33}$ element and coordinate structure (a), (b), (c) and (d) as well as coefficients of wavelet expansion $W_{a, b}$ (e), (f) for the $f_{33}$ element of $\mathrm{A}$ and $\mathrm{B}$ type connective tissues.

of optical axis orientations $\rho$ and values of phase shifts $\delta$ [2-8]. We considered the most spread case of pathological changes in BT architectonics-formation of directions for pathological growth or excrescence of a tumor. Within mathematical frames, this case was modeled as a superposition of statistical (equiprobable) and stochastic (quasiregular) components in distributions of orientations $\rho$ of birefringent fibrils as well as phase shifts $\delta$ that are caused by them

$$
\begin{aligned}
& Q_{1}(\rho) \approx R(\rho)+A \sin \frac{2 \pi}{D} \rho, \\
& Q_{2}(\delta) \approx R(\delta)+B \sin \frac{2 \pi}{D} \delta,
\end{aligned}
$$

where $Q_{i=1,2}$ are the functions of distributions for $\rho$ and $\delta$ values; $R$ is random (equiprobable) distribution of $\rho$ and $\delta$; $A, B$ are amplitudes of the stochastic component; $D$ is mean statistical size of co-axial fibrils.

The analysis of (3) shows the set of Mueller matrix elements presented by combinations "cos $\times \sin$ "; " $\cos ^{2}+$ $\sin ^{2} \times \cos$ "; " $\sin ^{2}+\cos ^{2} \times \cos$ ", which depend on $\rho$ and $\delta$.
That is why, one can assume that coordinate distributions $f_{i k}$, which characterize the real architectonics networks of biological tissues, are also presented by superposition of statistic and stochastic components.

For simplicity we consider one-dimensional coordinate distribution in the following form:

$$
f_{i k}(x)=R(x)+C \sin \frac{2 \pi}{D} x
$$

Here, $C$ is the amplitude of a stochastic component.

We modeled a superposition of a "background" $R(x)$ and informative signal $C \sin 2 \pi / D x$ for the following relations between their amplitudes $R(x)=0.01 \cdot C \sin (2 \pi / D) x \div 6$. $C \sin (2 \pi / D) x$.

Figure 1 shows wavelet coefficients $W_{a, b}$ for the respective distributions $f_{i k}(x)$.

As seen from the data obtained, the distributions of values for wavelet coefficients $W_{a, b}$ of all the types of signals $f_{i k}(x)$ behave like quasiharmonic structures. Even in the case 
TABLE 2: Log-log dependences of power spectra for the wavelet coefficients $W_{a, b}$ of statistical-stochastic distributions for the $f_{33}$ element of $\mathrm{A}$ and $\mathrm{B}$ type connective tissues.
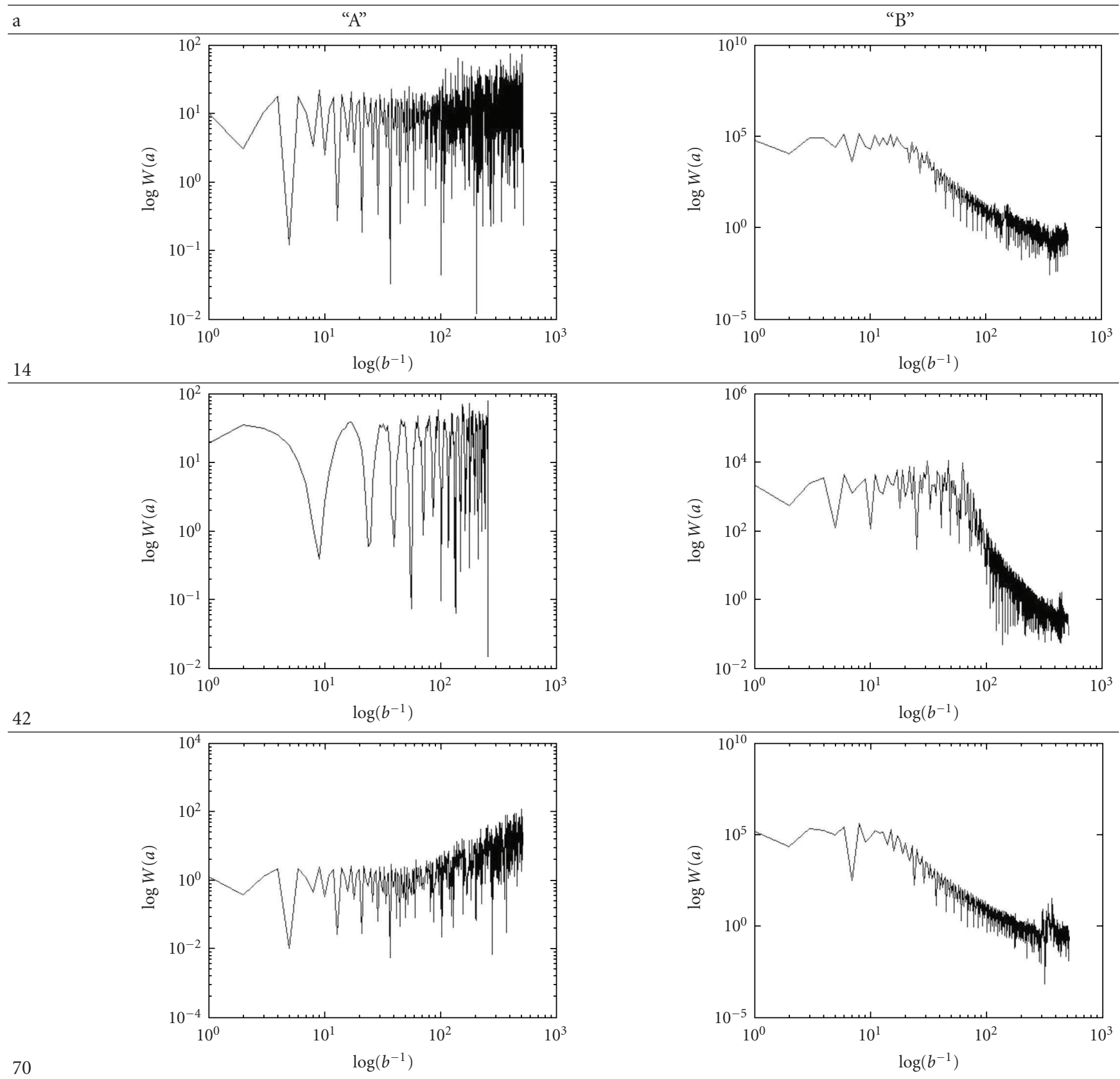

of significant (six-fold) dominance of the statistical component amplitude (Figure 1(c)), the quasiregular structure of coordinate distribution for wavelet coefficients $W_{a, b}$ is preserved in full. This fact confirms a high efficiency of the wavelet analysis in separation of the harmonic component in the distribution of $f_{i k}(x)$ elements of the Mueller matrix.

To make diagnostic possibilities of the wavelet analysis more objective, we calculated statistical moments of the first to fourth orders $(M, \sigma, A, E)$, which characterize distributions of the wavelet coefficients $W_{a, b}\left(f_{i k}\right)$ for various ratios $0.01 \leq A_{0} / A \leq 6$ (Figure 2), and found their Log-log dependences of power spectra (Table 1 ):

$$
\begin{gathered}
M=\frac{1}{m \times n} \sum_{i=1}^{m \times n}\left|W_{a, b}\left(f_{i k}\right)\right|, \\
\sigma=\sqrt{\frac{1}{m \times n} \sum_{i=1}^{m \times n}\left[W_{a, b}\left(f_{i k}\right)\right]^{2},} \\
A=\frac{1}{\sigma_{2}^{3}} \frac{1}{m \times n} \sum_{i=1}^{m \times n}\left[W_{a, b}\left(f_{i k}\right)\right]^{3}, \\
E=\frac{1}{\sigma_{2}^{4}} \frac{1}{m \times n} \sum_{i=1}^{m \times n}\left[W_{a, b}\left(f_{i k}\right)\right]^{4} .
\end{gathered}
$$




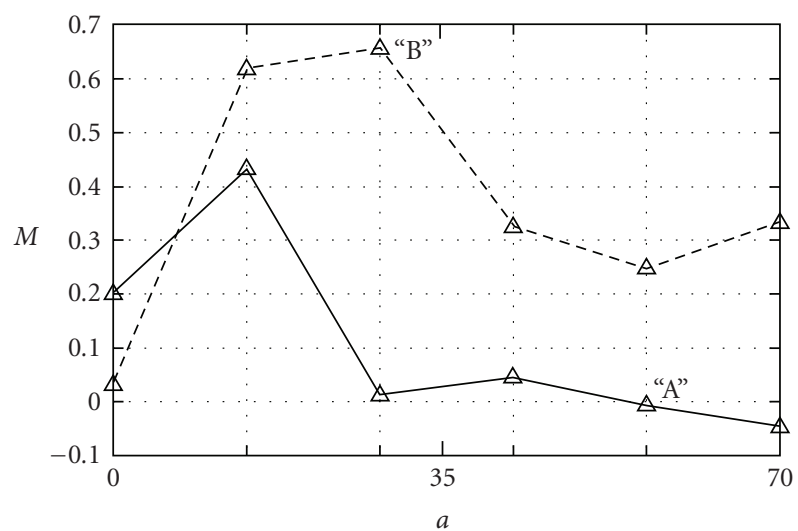

(a)

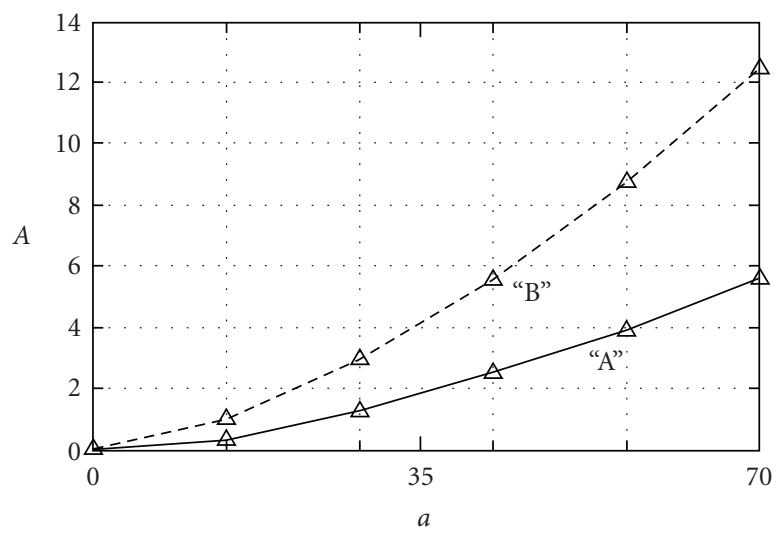

(c)

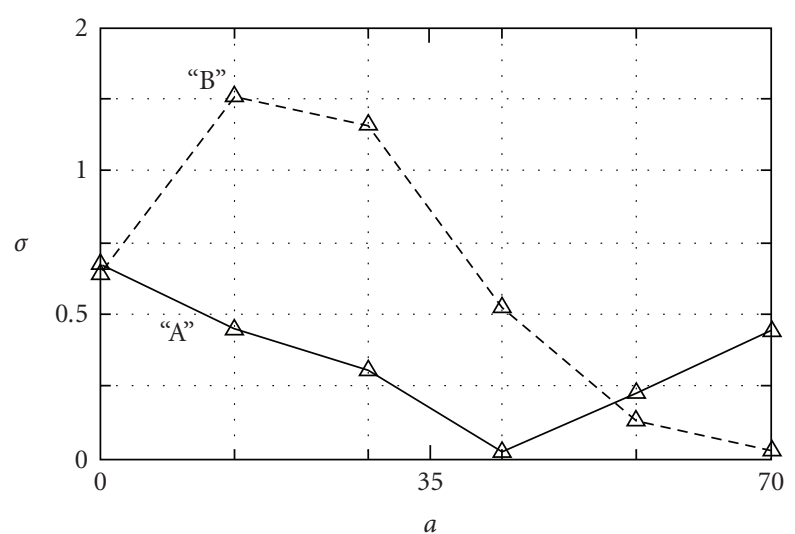

(b)

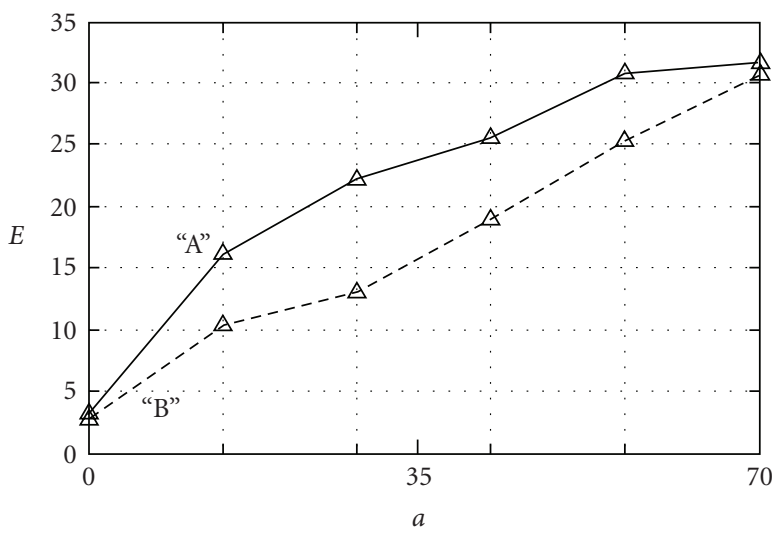

(d)

Figure 5: Dependences of statistical moments of the 1st to 4th orders on the scale of the wavelet function $\mu$ for connective tissue of A and B types.

Our analysis of the obtained data revealed that the change of the fourth statistical moment for the distribution of wavelet coefficients $W_{a, b}$ is the most dynamical from the above viewpoint, as the value of this moment changes within the range of one order in dependency of ratios $0.01 \leq$ $A_{0} / A \leq 6$.

Our investigation of log-log dependences for power spectra of distributions describing the wavelet coefficients $W_{a, b}$ of matrix elements $f_{i k}(x)$ allowed revealing the following regularities:

(i) all the dependences $\log W_{a, b}-\log (1 / d)$ calculated for various relations between amplitudes of random and quasi-regular components of distributions characteristic for the Mueller matrix elements $f_{i k}(x)$ consist of two parts, namely, the fractal one (with one slope of the approximating curve within a definite range $\Delta d$ for sizes of birefringent fibrils) and the statistical one (when a stable value for the slope angle of the approximating curve does not take place);

(ii) when the amplitude of the statistical component in the $f_{i k}(x)$ distribution grows, the range $\Delta d$ of a linear part in dependences $\log W_{a, b}-\log (1 / d)$ is decreased; (iii) fractal component of log-log dependences for the power spectra of wavelet coefficients $W_{a, b}$ is preserved even for significant (six-fold) dominance of the noise amplitude and comprises the size range $\Delta d$ $=50-100 \mu \mathrm{m}$.

Thus, the performed computer modeling indicates the diagnostic efficiency of the wavelet analysis when detecting local changes in birefringency $(\delta)$ of ordered biological crystals.

Besides, using the statistical and correlation analysis of wavelet coefficients $W_{a, b}$ in the expansion of the Mueller matrix elements, we have demonstrated the possibility to reveal the quasiharmonic component in distributions of orientation $(\rho)$ and phase $(\delta)$ parameters in complex (statistical) architectonic networks.

\section{Scheme and Methods of Experimental Investigations}

Figure 3 shows traditional optical scheme of polarimeter for measuring due to technique provided in [14] of the Mueller matrix elements of BT histological sections.

The parallel $\left(\varnothing=10^{4} \mu \mathrm{m}\right)$ beam of He-Ne laser $(\lambda$ $=0.6328 \mu \mathrm{m}, W=5.0 \mu \mathrm{W})$ was used as an illuminator. 
Polarization illuminator consists of quarter-wave plates 3, 5 and polarizer 4, and it sequentially forms a series of linearly polarized $\left(I_{0}, I_{45}, I_{90}, I_{135}\right)$ with azimuths $0^{\circ}, 90^{\circ}$, $45^{\circ}, 135^{\circ}$, and right-hand $\left(I_{\otimes}\right)$ and left-hand $\left(I_{\oplus}\right)$ circularly polarized probing BT laser beams. The BT images made by microobjective $(4 \times) 7$ were projected into the plane of a light-sensitive plate $(m \times n=800 \times 600$ pixels $)$ of CCD-camera 10. Polarization analysis of the BT images was performed by means of polarizer 9 and quarter-wave plate 8 .

The optical thin (the absorption coefficient $\tau<0,1$ ) BT histological sections were used as the objects of investigation. In this situation one has a single scattering regime of laser radiation scattered by BT network and the narrowband scattering indicatrix is formed (95\% of energy is concentrated within the angle cone $\Delta \Omega \leq 15^{\circ}$ ). Therefore, the speckle background formation in the BT histological section image due to scattering on optical elements is insignificant.

The technique of obtaining such objects is convenient: biological tissue is freezing to nitrogen temperature with the following obtaining, by means of medical microtome, the histological sections (from $10 \mu \mathrm{m}$ to $100 \mu \mathrm{m}$ ). Such a technique allows preserving the optical properties of biological tissues over a period of 24 hours.

\section{The Diagnostics of Local Changes in the Optical and Geometrical Structure of Architectonic Networks Inherent to Real Biological Tissues}

We performed comparative investigations of two types of mounts from connective tissue of a woman matrix:

(i) healthy tissue (type A) - the set of chaotically oriented collagen fibrils;

(ii) tissue in the state of dysplasia (precancer state (type B)) - the set of chaotically oriented collagen fibrils with local quasiordered parts.

From the optical viewpoint, polarization properties of these tissues (types A and B) are similar to some extent. For instance, the coordinate distribution of random values inherent to phase shifts $\delta(x, y)$, which is related with the range of changes in geometric sizes of collagen fibrils, is close in both cases. The main differences in composition of the set of biological crystals lie in presence of local parts with quasi-ordered directions of optical axes in the tissue of the type B. Being based on this fact, one can assume that the coordinate distribution of the Mueller matrix element (Mueller-matrix images-(MMIs) values for the type A tissue $f_{i k}(\rho, \delta)$ approaches the statistical one. The coordinate distribution $f_{i k}(\rho, \delta)$ for the type B tissue can be represented by a superposition of the random and quasiregular components (8).

As a main element of the Mueller matrix for biological tissue of a given type, we chose the "orientation" matrix element $f_{33}$. It is known that the statistical and correlation analysis of coordinate distributions for this element is considered as efficient in differentiation of optical properties characterizing collagen networks in healthy and pathologically changed skin [12].

Shown in Figure 4 are MMIs for the element $f_{33}$ (fragment (a): type A, fragment (b): type B) and the respective, wavelet expansions (fragments (e) and (f) for the linear cross-section of $f_{33}(x, y)$, fragments (c) and (d) for healthy and pathologically changed connective tissue of the uterus neck).

Our comparative analysis of the obtained data shows a complex statistical structure of two-dimensional distributions for the matrix element $f_{33}(x, y)$ (see fragments (a) and (b)) as well as its linear cross-sections (fragments (c) and (d)) for MMI of biological tissues for their both types. The same can be stated for the distributions of wavelet coefficients $W_{a, b}$ (fragments (e) and (f)).

With account of the above observations, it seems actual to verify the efficiency of statistical (the set of the first to fourth moments for $W_{a, b}$ distributions) and correlation (features of the power spectra for wavelet coefficients of $W_{a, b}$ ) methods of analysis for diagnostics of formation of local parts containing quasi-ordered $(\rho(x, y) \approx$ const $)$ birefringent fibrils.

With this aim, we performed step-by-step "screening" of the pictures for wavelet coefficients $W_{a, b}$ (Figures $4(\mathrm{e})$ and $4(\mathrm{f}))$ using the scale change for the wavelet function $\mu=$ 0.5 . For each value of the wavelet function $a_{i}$, we found the dependences $W\left(b, a=a_{i}\right)$ and calculated the log-log dependences for the scale of their power spectra. As a result of this linear scanning, we obtained the array of data $\left\{\log W\left(a_{1}\right)-\log (b)\right\} \div\left\{\log W\left(a_{j}\right)-\log (b)\right\}$ summarized in Table 2 .

Coordinate distributions for the matrix element $f_{33}$ corresponding to a mount of the sample of healthy connective tissue (Table 2) are characterized by statistical distributions for the wavelet coefficients $W_{a, b}$ in all the range of scale $a$ changes of the wavelet function $\mu$. It is confirmed by the absence of any stable slope of the approximating curve for the considered set of dependences $\log W_{a, b}(1 \leq a \leq$ $5 b)-\log \left(b^{-1}\right)$.

Another picture can be observed for the sample of changed connective tissue (Figure 4). If the scale coefficient $a$ of the wavelet function $\mu$ possesses some definite dimension, then there exists a linear part in the respective loglog dependence of the power spectrum characterizing the distribution of wavelet coefficients $W_{a, b}\left(a_{i}\right)$. In accordance with performed computer modeling, this fact indicates the availability of a quasi-harmonic component in $W_{a, b}\left(a_{i}\right)$ distributions, which is caused by respective geometry of biological crystals.

In our case, for the mean statistical size of a pathological creation $a=28 \mu \mathrm{m}$ one can observe a quasi-ordered part of collagen fibrils with sizes lying within the range 10 to $50 \mu \mathrm{m}$.

Thus, one can state that the correlation approach, in the analysis of $W_{a, b}$ coefficients valid for the wavelet distribution in MMI of "orientation" element $f_{33}$ for connective tissue of the uterus neck, is rather efficient for differentiation of its healthy and pathologically changed samples. 
Some additional information for differentiation of these objects was obtained using the statistical analysis of coordinate distributions $W_{a, b}\left(a_{i}\right)$.

Shown in Figure 5 are the dependences of statistical moments of the firest to fourth orders for coordinate distributions of wavelet coefficients $W_{a, b}$ in MMI of $f_{33}$, which correspond to different scales of the wavelet function $\mu$.

The obtained data show that

(i) statistical moments of the 1st and 4th orders for distributions of wavelet coefficients $W_{a, b}$, like of the case of computer modeling (Figure 2) the $f_{33}$ element describing the samples of myometrium of both types, suffer insignificant changes ( 0 to 0.2 ) within the whole range of scales $a_{i}$ for the wavelet function $\mu$ (Figure 5),

(ii) the range of changes in values of skewness $(A)$ and kurtosis $(E)$ for $W_{a, b}$ distributions lies within the range of two orders (Figure 5),

(iii) main differences between connective tissues of $\mathrm{A}$ and B types are found in the vicinity of the scale $a=$ $28 \mu \mathrm{m}$ where dependences between $A(a)$ and $E(a)$ reach two- or three-fold level.

Thus, we found that the differences between values of statistical moments of higher orders for a definite range of scales $a$ of the wavelet function $\mu_{a}$ can be also used to differentiate local changes in orientation changes of optical axes inherent to territorial matrix crystals.

\section{References}

[1] S. C. Cowin, "How is a tissue built?" Journal of Biomechanical Engineering, vol. 122, no. 6, pp. 553-569, 2000.

[2] O. V. Angelsky, A. G. Ushenko, Yu. A. Ushenko, Ye. G. Ushenko, Yu. Ya. Tomka, and V. P. Pishak, "Polarizationcorrelation mapping of biological tissue coherent images," Journal of Biomedical Optics, vol. 10, no. 6, Article ID 064025, 2005.

[3] J. F. de Boer and T. E. Milner, "Review of polarization sensitive optical coherence tomography and Stokes vector determination," Journal of Biomedical Optics, vol. 7, no. 3, pp. 359-371, 2002.

[4] J. F. de Boer, T. E. Milner, and J. S. Nelson, "Two dimensional birefringence imaging in biological tissue using phase and polarization sensitive optical coherence tomography," in Trends in Optics and Photonics (TOPS): Advances in Optical Imaging and Photon Migration, OSA, Washington, DC, USA, 1998.

[5] M. J. Everett, K. Schoenenberger, B. W. Colston, and L. B. Da Silva, "Birefringence characterization of biological tissue by use of optical coherence tomography," Optics Letters, vol. 23, no. 3, pp. 228-230, 1998.

[6] O. V. Angelsky, G. V. Demianovsky, A. G. Ushenko, D. N. Burkovets, and Yu. A. Ushenko, "Wavelet analysis of two-dimensional birefringence images of architectonics in biotissues for diagnosing pathological changes," Journal of Biomedical Optics, vol. 9, no. 4, pp. 679-690, 2004.

[7] O. V. Angelsky, Yu. Ya. Tomka, A. G. Ushenko, Ye. G. Ushenko, S. B. Yermolenko, and Yu. A. Ushenko, "2-D tomography of biotissues images in pre-clinic diagnostics of their pre-cancer states," in Advanced Topics in Optoelectronics, Microelectronics, and Nanotechnologies II, vol. 5972 of Proceedings of the SPIE, pp. 158-162, 2004.

[8] O. V. Angelsky, A. G. Ushenko, D. N. Burkovets, and Yu. A. Ushenko, "Polarization visualization and selection of biotissue image two-layer scattering medium," Journal of Biomedical Optics, vol. 10, no. 1, Article ID 014010, 2005.

[9] O. V. Angelsky, Yu. Ya. Tomka, A. G. Ushenko, Ye. G. Ushenko, and Yu. A. Ushenko, "Investigation of 2D Mueller matrix structure of biological tissues for pre-clinical diagnostics of their pathological states," Journal of Physics D, vol. 38, no. 23, pp. 4227-4235, 2005.

[10] S. Jiao, W. Yu, G. Stoica, and L. V. Wang, "Optical-fiber-based Mueller optical coherence tomography," Optics Letters, vol. 28, no. 14, pp. 1206-1208, 2003.

[11] Yu. A. Ushenko, "Statistical structure of polarizationinhomogeneous images of biotissues with different morphological structures," Ukrainian Journal of Physical Optics, vol. 6, pp. 63-70, 2005.

[12] O. V. Angelsky, A. G. Ushenko, Yu. A. Ushenko, Ye. G. Ushenko, Yu. Ya. Tomka, and V. P. Pishak, "Polarizationcorrelation mapping of biological tissue coherent images," Journal of Biomedical Optics, vol. 10, no. 6, Article ID 064025, 2005.

[13] O. V. Angelsky, A. G. Ushenko, and Yu. A. Ushenko, "Polarization reconstruction of orientation structure of biological tissues birefringent architectonic nets by using their Muellermatrix speckle-images," Journal of Holography and Speckle, vol. 2, pp. 72-79, 2005.

[14] A. G. Ushenko and V. P. Pishak, "Laser polarimetry of biological tissue. Principles and applications," in Coherent-Domain Optical Methods. Biomedical Diagnostics, Environmental and Material Science, V. Tuchin, Ed., pp. 67-93, Kluwer Academic Publishers, Dordrecht, The Netherlands, 2004.

[15] O. V. Angelsky, A. G. Ushenko, D. M. Burkovets, V. P. Pishak, and O. V. Pishak, "Laser polarimetry of pathological changes in biotissues," in The 7th International Symposium on Laser Metrology Applied to Science, Industry, and Everyday Life, vol. 4900 of Proceedings of the SPIE, pp. 1045-1049, 2002.

[16] O. V. Angelsky, D. N. Burkovets, A. V. Kovalchuk, and S. G. Hanson, "Fractal description of rough surfaces," Applied Optics, vol. 41, no. 22, pp. 4620-4629, 2002.

[17] O. V. Angelsky, P. P. Maksimyak, and T. O. Perun, "Optical correlation method for measuring spatial complexity in optical fields," Optics Letters, vol. 18, no. 2, pp. 90-92, 1993.

[18] O. V. Angelsky, D. N. Burkovets, P. P. Maksimyak, and S. G. Hanson, "Applicability of the singular-optics concept for diagnostics of random and fractal rough surfaces," Applied Optics, vol. 42, no. 22, pp. 4529-4540, 2003.

[19] O. V. Angelsky, P. P. Maksimyak, and T. O. Perun, "Dimensionality in optical fields and signals," Applied Optics, vol. 32, no. 30, pp. 6066-6071, 1993. 

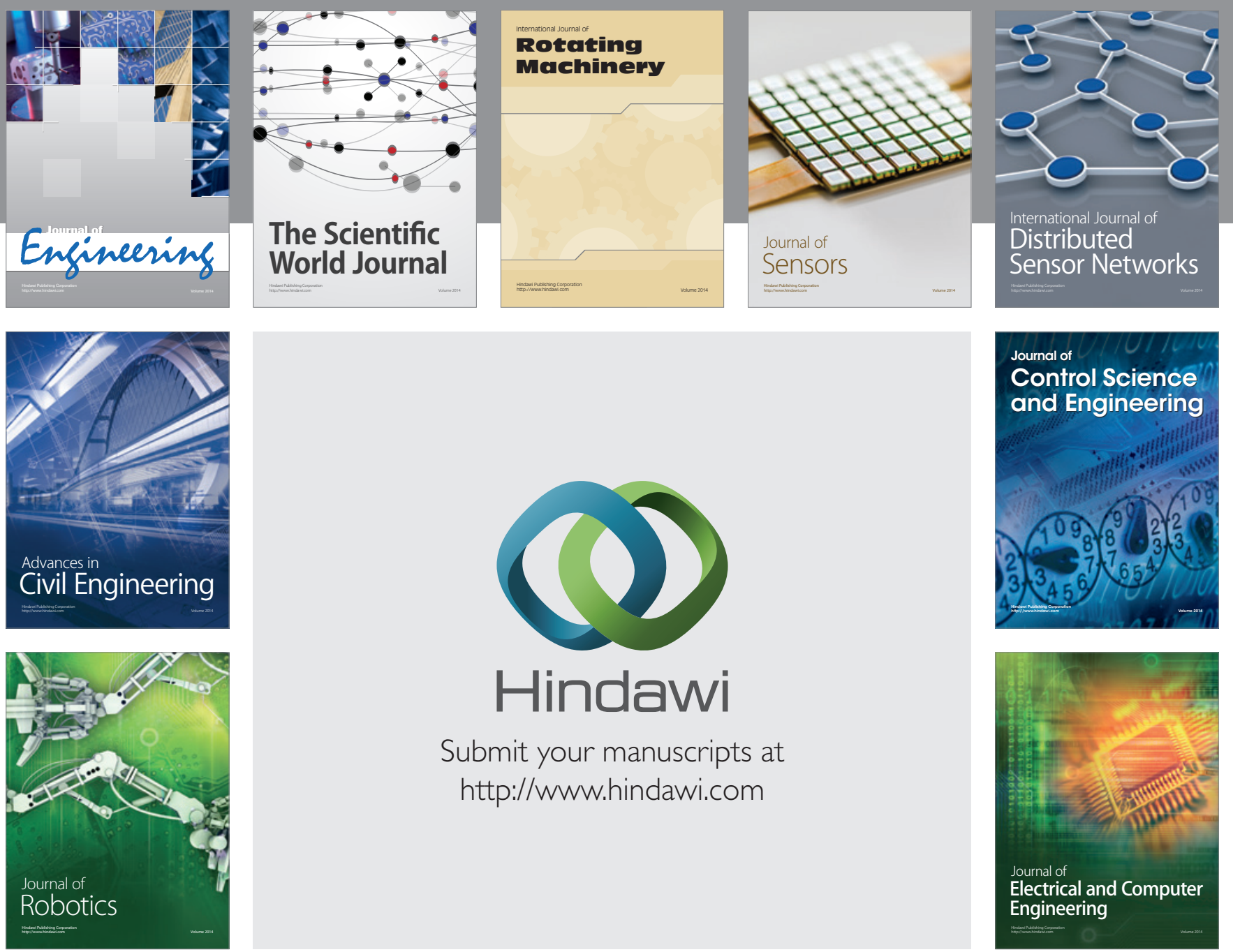

Submit your manuscripts at

http://www.hindawi.com
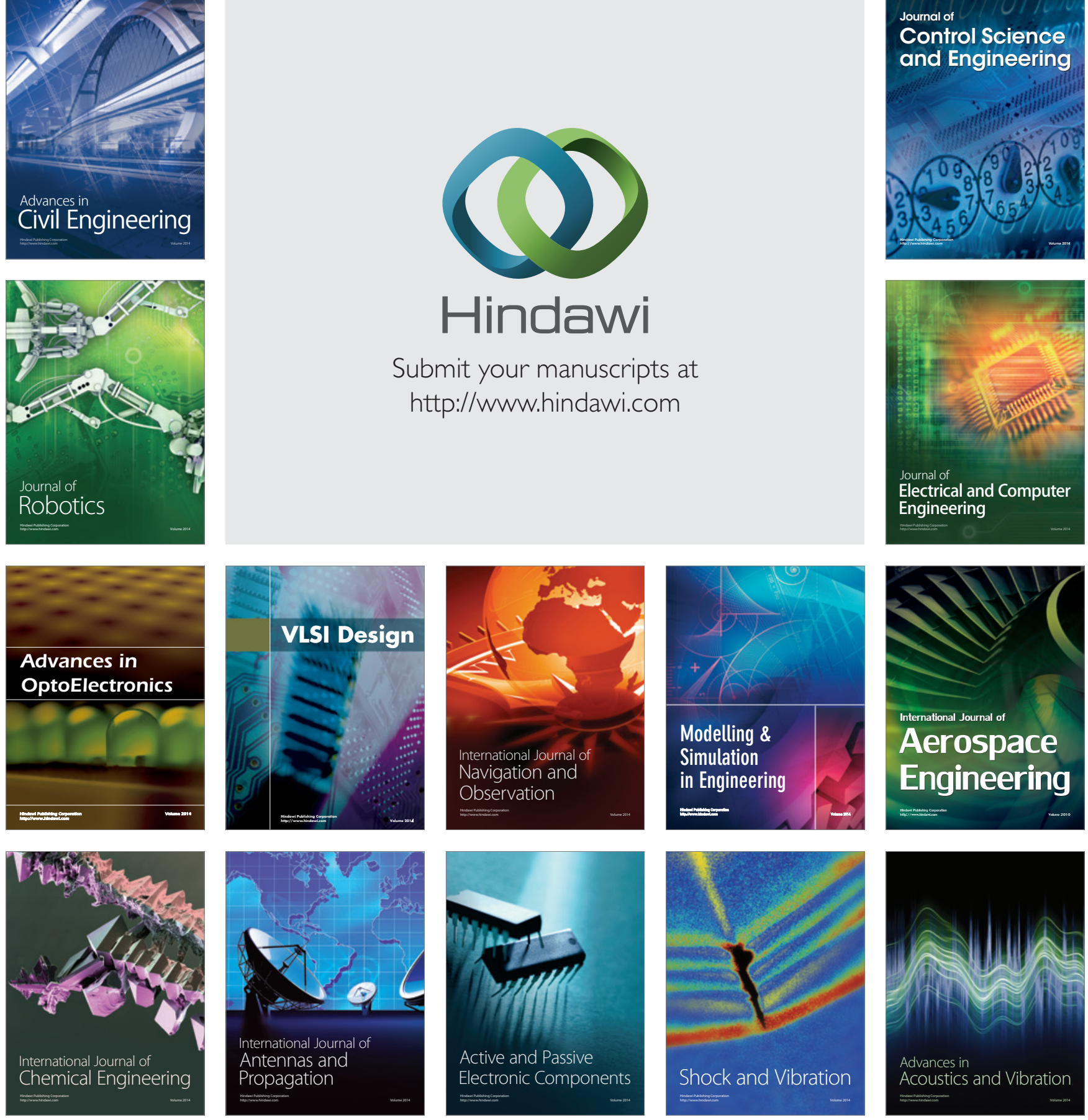